\title{
Chromosome Fragile Site
}

National Cancer Institute

\section{Source}

National Cancer Institute. Chromosome Fragile Site. NCI Thesaurus. Code C13375.

Heritable sensitive regions of chromosomes which show up in vitro as non-staining bands. They are associated with chromosome breakage and other aberrations. No abnormal phenotype has been definitely identified with autosomal fragile sites, but some rare autosomal recessive disorders may be due to homozygosity for fragile sites. A fragile site on the $X$ chromosome is associated with Fragile $X$ Syndrome. Fragile sites are designated by the letters "fra" followed by the designation for the specific chromosome and locus. 